\title{
Effect of the Coronavirus Disease 2019 Pandemic on the Quality of Stroke Care in Stroke Units and Alternative Wards: A National Comparative Analysis
}

\author{
Dominique A. Cadilhac, ${ }^{\mathrm{a}, \mathrm{b}}$ Joosup Kim, ${ }^{\mathrm{a}, \mathrm{b}}$ Geoffrey Cloud, ${ }^{\mathrm{c}, \mathrm{d}}$ Craig S. Anderson, ${ }^{\mathrm{e}}$ Emma K. Tod, ${ }^{\mathrm{b}}$ \\ Sibilah J. Breen, ${ }^{\mathrm{b}}$ Steven Faux, ${ }^{\mathrm{f}, \mathrm{g}}$ Timothy Kleinig, ${ }^{\mathrm{h}}$ Helen Castley, ${ }^{\mathrm{i}}$ Richard I. Lindley, ${ }^{\mathrm{j}}$ \\ Sandy Middleton, ${ }^{\mathrm{k}}$ Bernard Yan, ${ }^{1}$ Kelvin Hill, ${ }^{\mathrm{m}}$ Brett Jones, ${ }^{\mathrm{n}}$ Darshan Shah, ${ }^{\mathrm{o}, \mathrm{p}}$ Katherine Jaques, ${ }^{\mathrm{q}}$ \\ Benjamin Clissold, ${ }^{\mathrm{a}, \mathrm{r}}$ Bruce Campbell, ${ }^{\mathrm{b}, \mathrm{l}}$ Natasha A. Lannin, ${ }^{\mathrm{c}, \mathrm{d}}$ on Behalf of the AuSCR COVID-19 \\ Reporting Consortium Group \\ ${ }^{a}$ Department of Medicine, School of Clinical Sciences at Monash Health, Monash University, Clayton, Australia

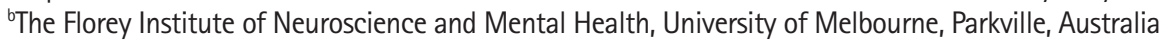 \\ 'Department of Neuroscience, Central Clinical School, Monash University, Clayton, Australia \\ 'Stroke Services, Alfred Health, Melbourne, Australia \\ 'The George Institute for Global Health, Sydney, Australia \\ fDepartment of Rehabilitation Medicine, St Vincent's Hospital Sydney, Sydney, Australia \\ ${ }^{9}$ Department of Clinical Medicine, St Vincent's Clinical School, University of New South Wales, Darlinghurst, Australia \\ hDepartment of Neurology, Royal Adelaide Hospital, Adelaide, Australia \\ 'Department of Neurology, Royal Hobart Hospital, Hobart, Australia \\ 'Department of Medicine, University of Sydney, Sydney, Australia \\ ${ }^{k}$ Nursing Research Institute, Australian Catholic University, Sydney, Australia \\ 'Department of Medicine and Neurology, Melbourne Brain Centre at the Royal Melbourne Hospital, University of Melbourne, Parkville, Australia \\ mStroke Foundation, Melbourne, Australia \\ "Department of Neurology, Canberra Hospital, Canberra, Australia \\ ${ }^{\circ}$ Department of Neurology, Gold Coast Hospital and Health Service, Southport, Australia \\ ${ }^{\mathrm{P}}$ Department of Neurology, Mater Brisbane, South Brisbane, Australia \\ "Stroke Statewide Clinical Network, Healthcare Improvement Unit, Clinical Excellence, Queensland Health, Brisbane, Australia \\ 'Department of Neurology, Monash Health, Clayton, Australia
}

Background and Purpose Changes to hospital systems were implemented from March 2020 in Australia in response to the coronavirus disease 2019 pandemic, including decreased resources allocated to stroke units. We investigate changes in the quality of acute care for patients with stroke or transient ischemic attack during the pandemic according to patients' treatment setting (stroke unit or alternate ward).

Methods We conducted a retrospective cohort study of patients admitted with stroke or transient ischemic attack between January 2019 and June 2020 in the Australian Stroke Clinical Registry (AuSCR). The AuSCR monitors patients' treatment setting, provision of allied health and nursing interventions, prescription of secondary prevention medications, and discharge destination. Weekly trends in the quality of care before and during the pandemic period were assessed using interrupted time series analyses.

Results In total, 18,662 patients in 2019 and 8,850 patients in 2020 were included. Overall, 75\% were treated in stroke units. Before the pandemic, treatment in a stroke unit was superior to alter-
Correspondence: Dominique A. Cadilhac School of Clinical Sciences at Monash Health, Monash University, Level 3 Hudson Institute Building, 27-31 Wright Street, Clayton VIC 3168, Australia Tel: +61-3-8572-2657 Fax: +61-3-9902-4245 E-mail:dominique.cadilhac@monash. edu https://orcid.org/0000-0001-8162-682X

Received: July 16, 2021 Revised: September 16, 2021 Accepted: September 17, 2021 
nate wards for the provision of all evidence-based therapies assessed. During the pandemic period, the proportion of patients receiving a swallow screen or assessment, being discharged to rehabilitation, and being prescribed secondary prevention medications decreased by $0.58 \%$ to $1.08 \%$ per week in patients treated in other ward settings relative to patients treated in stroke units. This change represented a $9 \%$ to $17 \%$ increase in the care gap between these treatment settings during the period of the pandemic that was evaluated ( 16 weeks).

Conclusions During the first 6 months of the pandemic, widening care disparities between stroke units and alternate wards have occurred.

Keywords Quality of health care; Stroke; COVID-19

\section{Introduction}

Hospital systems have been changed in response to the coronavirus disease 2019 (COVID-19) pandemic in order to ensure the virus does not spread, and that health care workers and visitors are kept safe. A consequence has been the redeployment of hospital staff, changes to emergency department workflows, cancelation of elective surgery, and repurposing of hospital wards to increase capacity. ${ }^{1}$ For conditions such as acute stroke or transient ischemic attack, reports on the effect of these disruptions have been mixed, but generally point to delays in presentation and treatment. ${ }^{2}$ Particularly of concern in Australia, is the fact that resources in hospitals with specialist services dedicated for stroke-whereby hospital beds are collocated and there is a dedicated and experienced interdisciplinary team that manages the patients in this part of the hospital (also known as a stroke unit ward)-have had the number of beds reduced or specialized staff redeployed. ${ }^{3}$ Care in organized, dedicated stroke units by expert, interdisciplinary clinicians ensures best-practice treatments are provided and outcomes are better for patients than if treated in alternate ward settings. ${ }^{4,5}$ We have previously reported that, despite few COVID-19 cases in the first wave of the pandemic (first case recorded January 25, 2020 until mid-June 2020, when new cases were under 20 per day), significantly fewer patients had access to stroke units, and the length of stay in the hospital decreased. ${ }^{6}$

The downstream effects of these changes in hospital treatment pathways for patients with stroke and transient ischemic attack during the pandemic have not been previously reported by the type of ward setting the patients have been managed within. We aimed to examine the quality of care for patients admitted to the hospital with stroke or transient ischemic attack during the COVID-19 pandemic according to the ward setting they were treated in (stroke units or other wards).

\section{Methods}

The data and study materials that support the findings of this study are available from the corresponding author with the permission of the Florey Institute of Neuroscience and Mental Health acting on behalf of the Australian Stroke Clinical Registry (AuSCR) Consortium. Restrictions apply to the availability of these data, which were used under license for this study (Requests to access these datasets should be directed to admin@ auscr.com.au).

The AuSCR is a clinical quality registry that was established in 2009 to monitor and improve the quality of care provided to patients admitted with acute stroke or transient ischemic attack. Public hospitals from six Australian states and territories contribute data to the AuSCR, with funding provided primarily by state governments. Hospitals participating in the AuSCR prospectively collect a standardized minimum dataset that includes information on demographic and clinical characteristics of patients, and evidence-based therapies provided during the admission and at discharge from the hospital. ${ }^{7}$ The evidence-based therapies monitored in the AuSCR include treatment in a stroke unit, the provision of reperfusion therapies (intravenous thrombolysis and endovascular clot retrieval), allied health and nursing interventions, the prescription of secondary stroke prevention medications, and provision of discharge care plans.

In this study, data on admissions between January 1, 2019 and June 23, 2020 were retrospectively analyzed. Only admissions from hospitals contributing data in both 2019 and 2020 were included in this analysis. Data were excluded from one hospital contributing fewer than 100 cases over the study period. The last 2 weeks of data for the study period were removed due to low case numbers entered into the AuSCR (defined as more than two standard deviations below the average number of admissions per week) because of delays with data collection (Supplementary Figure 1). 
This study was approved by the Monash University Human Research Ethics Committee (project 26558). Written informed consent for participation was not required for this study in accordance with the national legislation and the institutional requirements. The AuSCR uses opt-out consent and a waiver of consent for deaths in order to minimize selection bias. ${ }^{8}$

\section{Data analysis and statistics}

Descriptive statistics appropriate to the type and distribution of the data were used. Patient demographics, clinical characteristics, process of care metrics for the provision of evidence-based therapies, and hospital outcomes such as length of stay and discharge destination were compared between treatment groups and time periods using chi-square and Kruskal-Wallis tests. In the case where a specific evidence-based therapy for any individual hospital contained $>30 \%$ missing data, these hospitals were excluded from the analysis of that specific variable. In all other cases where there was missing data related to the provision of evidence-based therapies, it was assumed the therapy was not provided.

Proportions of patients provided allied health and nursing interventions (antithrombotic medications within 48 hours of arrival, mobilized, and swallow screen or assessment) and discharge processes (prescribed secondary prevention antihypertensive medication, antithrombotic medication and lipid lowering medication; discharged to the community with a care plan; and discharge to rehabilitation) were calculated by week and ward setting (stroke units or other wards). Differences between ward settings were assessed in an interrupted time series analyses. ${ }^{9}$ The provision of reperfusion therapies was not investigated in this analysis because provision of reperfusion therapies generally occurs in Emergency Departments and patients who receive reperfusion therapies are subsequently managed in a stroke unit since they require intensive monitoring.

Trends in the proportion of patients provided these evidence-based therapies were compared before and after the week including March 1, 2020 (week 61 in the model spanning February 25, 2020 to March 2, 2020). This interruption date of March 1, 2020 coincided with the first COVID-19-related death in Australia. Nationwide restrictions related to reducing COVID-19 transmission in the community were imposed on March 21, 2020. Seasonality in admissions was considered by using a lag period of 53 weeks for correlations. Estimates were adjusted by the number of episodes per week and the number of patients with different clinical diagnoses (stroke, intracerebral hemorrhage, transient ischemic attack, and undetermined stroke). Analyses related to the provision of antithrombotic medications and lipid lowering medication were conducted with patients with intracerebral hemorrhage excluded.

$\beta$ Coefficients related to the interrupted time series analyses signify either (1) the difference in the average weekly percentage change over several weeks (trend) between groups; or (2) the difference in a percentage change in a given week between groups. Positive values indicate a greater relative percentage increase in stroke units compared with other ward settings. Negative values indicate a greater relative percentage decrease in stroke units compared with other ward settings. Data were analyzed using STATA/SE 15.0 (StataCorp., College Station, TX, USA).

\section{Results}

There were 27,512 admissions from 61 hospitals (from five states and territories) registered in the AuSCR between January 2019 and June 2020 that were included for analysis (Table 1). The proportion of patients treated in a stroke unit decreased from $76 \%$ $(16,666$ of 21,892 admissions) during the pre-pandemic period to $69 \%$ (3,870 of 5,620 admissions) during the pandemic period $(P<0.001)$. Distributions of diagnosis, previous history of stroke, arrival by ambulance, and transfer from another hospital were different between the pre-pandemic and pandemic periods. Compared with patients who were treated outside of stroke units, patients treated in stroke units were more often male, more often had an ischemic stroke, less often had an in-hospital stroke, more often arrived by ambulance, more often transferred from another hospital, less often able to walk on admission (proxy for greater stroke severity), and had longer lengths of stay in both the pre-pandemic and pandemic periods.

Patients treated in stroke units more often received evidence-based care in both the pre-pandemic and pandemic periods compared with those treated in other ward settings (Table 2). Apart from discharge to inpatient rehabilitation, there were differences in the proportion of patients provided evidence-based therapies between the pre-pandemic and pandemic periods. Among those treated outside of stroke units, there was a reduction from the pre-pandemic period to the pandemic period in the proportion of patients receiving mobilization, swallow screen or assessment, and secondary prevention medications at discharge from the hospital. These changes were not observed in patients who were treated in stroke units.

\section{Trends in the provision of nursing and allied health interventions}

In the pre-pandemic period, there were small differences observed in the trends of provision of some nursing and allied health interventions between patients treated in stroke units and those treated outside of stroke units (Figure 1, Supplemen- 
Table 1. Patient characteristics by ward setting and pandemic period

\begin{tabular}{|c|c|c|c|c|c|c|c|}
\hline \multirow{3}{*}{ Characteristic } & \multirow{2}{*}{\multicolumn{3}{|c|}{$\begin{array}{c}\text { Pre-pandemic (Jan 1, 2019-Feb 24, 2020) } \\
\text { Treated in a stroke unit }\end{array}$}} & \multirow{2}{*}{\multicolumn{3}{|c|}{$\begin{array}{c}\text { Pandemic (Feb 25, 2020-Jun 23, 2020) } \\
\text { Treated in a stroke unit }\end{array}$}} & \multirow{3}{*}{$\begin{array}{c}\text { Comparison } \\
\text { of periods* } \\
P\end{array}$} \\
\hline & & & & & & & \\
\hline & No $(n=5,226)$ & Yes $(n=16,666)$ & $P$ & No $(n=1,750)$ & Yes $(n=3,870)$ & $P$ & \\
\hline Age (yr) & & & 0.083 & & & 0.062 & 0.643 \\
\hline$<65$ & $1,342(26)$ & $4,198(26)$ & & $404(23)$ & $1,009(26)$ & & \\
\hline $65-74$ & $1,236(24)$ & $3,935(24)$ & & $433(25)$ & $955(25)$ & & \\
\hline $75-84$ & $1,438(28)$ & $4,951(30)$ & & $523(30)$ & $1,130(29)$ & & \\
\hline$\geq 85$ & $1,051(21)$ & $3,283(20)$ & & $373(22)$ & 741 (19) & & \\
\hline Male & $2,635(52)$ & $9,322(57)$ & $<0.001$ & $924(53)$ & $2,162(56)$ & 0.034 & 0.636 \\
\hline Diagnosis & & & $<0.001$ & & & $<0.001$ & 0.001 \\
\hline Intracerebral hemorrhage & 794 (16) & $1,786(11)$ & & 214 (15) & $443(12)$ & & \\
\hline Ischemic stroke & 2,387 (48) & $12,781(77)$ & & $671(47)$ & $2,885(75)$ & & \\
\hline Transient ischemic attack & $1,633(33)$ & $1,807(11)$ & & $490(34)$ & $455(12)$ & & \\
\hline Undetermined stroke & $194(4)$ & $236(1)$ & & $60(4)$ & $50(1)$ & & \\
\hline In-hospital stroke & $353(7)$ & $433(3)$ & $<0.001$ & $77(6)$ & $92(2)$ & $<0.001$ & 0.367 \\
\hline Previous history of stroke & $1,029(21)$ & $3,636(22)$ & 0.150 & 214 (19) & $743(21)$ & 0.105 & 0.012 \\
\hline Arrival by ambulance & $3,346(73)$ & $12,478(78)$ & $<0.001$ & $913(77)$ & $2,913(81)$ & 0.002 & $<0.001$ \\
\hline Transferred from another hospital & $547(11)$ & $3,139(19)$ & $<0.001$ & $118(8)$ & 656 (17) & $<0.001$ & $<0.001$ \\
\hline Ability to walk on admission & $2,181(48)$ & $6,432(41)$ & $<0.001$ & 496 (47) & $1,384(40)$ & $<0.001$ & 0.298 \\
\hline $\begin{array}{l}\text { Median length of stay (day) } \\
\text { (quartile 1-3) }\end{array}$ & $2(1-5)$ & $4(2-8)$ & $<0.001$ & $2(1-5)$ & $4(2-8)$ & $<0.001$ & $<0.001$ \\
\hline
\end{tabular}

Values are presented as number (\%) unless otherwise specified.

*Patients within periods were aggregated in this comparison.

Table 2. Treatment by ward setting and pandemic period

\begin{tabular}{|c|c|c|c|c|c|c|c|}
\hline \multirow{3}{*}{ Variable } & \multirow{2}{*}{\multicolumn{3}{|c|}{$\begin{array}{c}\text { Pre-pandemic (Jan 1, 2019-Feb 24, 2020) } \\
\text { Treated in a stroke unit }\end{array}$}} & \multirow{2}{*}{\multicolumn{3}{|c|}{$\begin{array}{c}\text { Pandemic (Feb 25, 2020-Jun 23, 2020) } \\
\text { Treated in a stroke unit }\end{array}$}} & \multirow{3}{*}{$\begin{array}{c}\text { Comparison } \\
\text { of periods* }\end{array}$} \\
\hline & & & & & & & \\
\hline & No & Yes & $P$ & No & Yes & $P$ & \\
\hline $\begin{array}{l}\text { Provided antithrombotic } \\
\text { medication within } 48 \text { hours } \\
\text { of arrival }^{\dagger}\end{array}$ & $\begin{array}{l}2,092 / 3,371 \\
(62)\end{array}$ & $\begin{array}{l}8,289 / 11,697 \\
(71)\end{array}$ & $<0.001$ & $\begin{array}{c}543 / 843 \\
(64)\end{array}$ & $\begin{array}{l}1,840 / 2,484 \\
(74)\end{array}$ & $<0.001$ & 0.002 \\
\hline Mobilised during the admission & $\begin{array}{c}3,113 / 4,951 \\
(63)\end{array}$ & $\begin{array}{c}11,679 / 14,692 \\
(79)\end{array}$ & $<0.001$ & $\begin{array}{l}719 / 1,578 \\
(46)\end{array}$ & $\begin{array}{l}2,412 / 3,402 \\
(71)\end{array}$ & $<0.001$ & $<0.001$ \\
\hline $\begin{array}{l}\text { Provided swallow screen } \\
\text { or assessment }\end{array}$ & $\begin{array}{l}2,478 / 4,631 \\
(54)\end{array}$ & $\begin{array}{c}12,697 / 14,129 \\
(90)\end{array}$ & $<0.001$ & $\begin{array}{c}583 / 1,385 \\
(42)\end{array}$ & $\begin{array}{l}2,709 / 3,029 \\
(89)\end{array}$ & $<0.001$ & $<0.001$ \\
\hline $\begin{array}{l}\text { Discharged to inpatient } \\
\text { rehabilitation }\end{array}$ & $\begin{array}{l}506 / 5,012 \\
(10)\end{array}$ & $\begin{array}{l}4,234 / 16,249 \\
(26)\end{array}$ & $<0.001$ & $\begin{array}{l}155 / 1,330 \\
(12)\end{array}$ & $\begin{array}{l}987 / 3,578 \\
\quad(28)\end{array}$ & $<0.001$ & 0.141 \\
\hline $\begin{array}{l}\text { Prescribed antihypertensive } \\
\text { medication at discharge }\end{array}$ & $\begin{array}{l}2,656 / 4,059 \\
(65)\end{array}$ & $\begin{array}{l}11,159 / 14,607 \\
(76)\end{array}$ & $<0.001$ & $\begin{array}{l}613 / 1,245 \\
(49)\end{array}$ & $\begin{array}{l}2,352 / 3,260 \\
(72)\end{array}$ & $<0.001$ & $<0.001$ \\
\hline $\begin{array}{l}\text { Prescribed antithrombotic } \\
\text { medication at discharge }{ }^{+}\end{array}$ & $\begin{array}{c}3,157 / 3,773 \\
(84)\end{array}$ & $\begin{array}{c}12,642 / 13,636 \\
(93)\end{array}$ & $<0.001$ & $\begin{array}{l}724 / 1,175 \\
(62)\end{array}$ & $\begin{array}{c}2,626 / 3,022 \\
(87)\end{array}$ & $<0.001$ & $<0.001$ \\
\hline $\begin{array}{l}\text { Prescribed lipid lowering } \\
\text { medication at discharge }^{+}\end{array}$ & $\begin{array}{l}2,587 / 3,769 \\
(69)\end{array}$ & $\begin{array}{c}11,039 / 13,649 \\
(81)\end{array}$ & $<0.001$ & $\begin{array}{c}616 / 1,177 \\
(52)\end{array}$ & $\begin{array}{c}2,268 / 3,004 \\
(76)\end{array}$ & $<0.001$ & $<0.001$ \\
\hline $\begin{array}{l}\text { Discharged to the community } \\
\text { with a care plan }\end{array}$ & $\begin{array}{l}1,303 / 2,656 \\
(49)\end{array}$ & $\begin{array}{l}5,576 / 7,499 \\
(74)\end{array}$ & $<0.001$ & $\begin{array}{l}322 / 624 \\
(52)\end{array}$ & $\begin{array}{l}1,284 / 1,606 \\
(80)\end{array}$ & $<0.001$ & $<0.001$ \\
\hline
\end{tabular}

Values are presented as number/total number (\%).

*Patients within periods were aggregated in this comparison; ${ }^{+}$Excluding patients with intracerebral hemorrhage. 


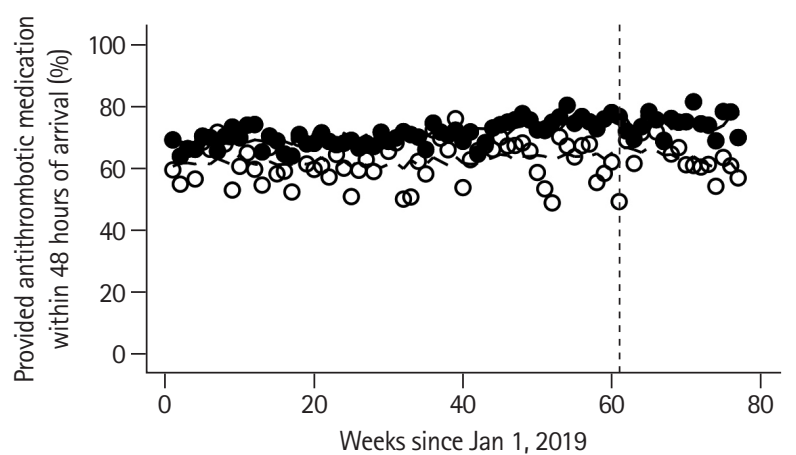

A

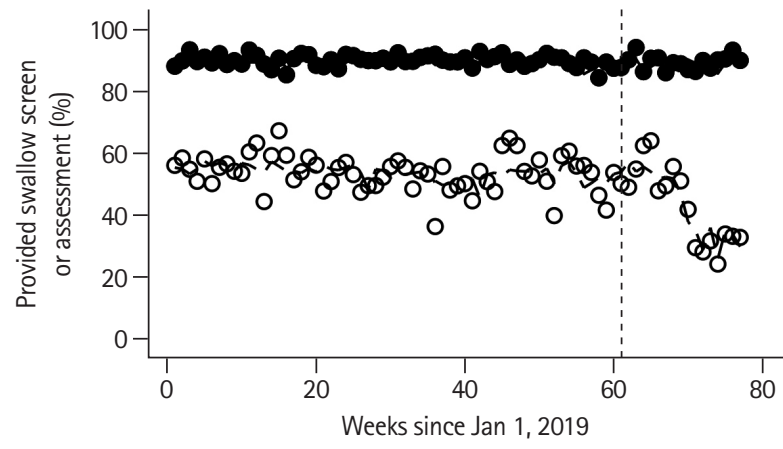

C

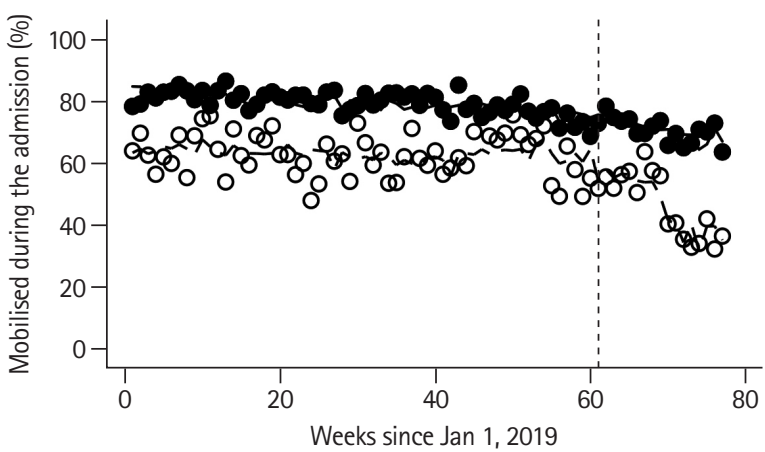

B

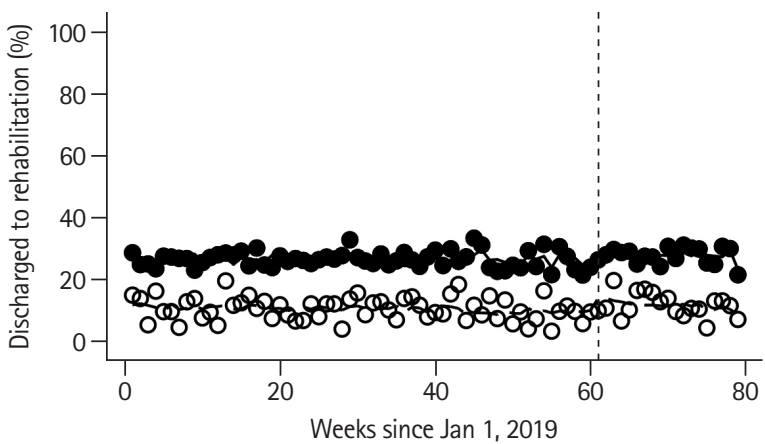

(D)

Treated in a stroke unit:

Treated in other ward setting:

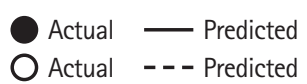

Figure 1. Proportion of patients (A) provided antithrombotic medication within 48 hours of arrival (excluding patients with intracerebral hemorrhage), (B) mobilized during the admission, (C) provided swallow screen or assessment, and (D) discharged to rehabilitation. The vertical line (week 61) indicates the week of the interruption used in this study (1/3/2020, the first coronavirus disease 2019 [COVID-19] related death in Australia). Significant difference in weekly trend between groups prior to interruption ( $\beta$ and 95\% confidence intervals [Cls] presented): provided antithrombotic medication within 48 hours of arrival 0.09 (95\% Cl, 0.04 to 0.14); mobilized during the admission $-0.15(95 \% \mathrm{Cl},-0.23$ to -0.07$)$. Significant difference during week of interruption between groups (week 61): provided antithrombotic medication within 48 hours of arrival $-5.35(95 \% \mathrm{Cl},-9.08$ to -1.61$)$; provided swallow screen or assessment -5.81 (95\% $\mathrm{Cl}_{1}-8.62$ to -2.99$)$; discharged to rehabilitation $-2.2(95 \% \mathrm{Cl},-3.72$ to -0.68$)$. Significant difference in weekly trend between groups in post-interruption period: provided swallow screen or assessment $0.86(95 \% \mathrm{Cl}, 0.48$ to 1.24$)$; discharged to rehabilitation $0.6(95 \% \mathrm{Cl}, 0.37$ to 0.83$)$. $\beta$ in a period signifies the difference in the average weekly percentage change over several weeks (trend) between groups. $\beta$ during the week of the interruption signifies the difference in a percentage change during that week between groups. Positive values indicate a greater relative percentage increase in the provision of evidence-based care in stroke units compared with other ward settings. Negative values indicate a greater relative percentage decrease in the provision of evidence-based care in stroke units compared with other ward settings. Other point estimates relevant to the figure are provided in the Supplementary Table 1.

tary Table 1). There was a 0.09\% per week increase in the provision of antithrombotic medications within 48 hours of arrival in patients treated in stroke units relative to those treated outside of a stroke unit $(\beta=0.09 ; 95 \%$ confidence interval $[\mathrm{Cl}]$, 0.04 to 0.14$)$, and a $0.15 \%$ per week decrease in mobilization during the admission in patients treated in stroke units relative to those treated outside of a stroke unit $(\beta=-0.15 ; 95 \% \mathrm{Cl}$, -0.23 to -0.07$)$. No such differences in trends were detected within and between groups for the provision of swallow screen or assessment and discharge to rehabilitation during the pre-pandemic period.

During the first week of the pandemic period, there was a relative decrease in the proportion of patients provided nursing and allied health interventions among patients treated in stroke units compared with those treated outside of stroke units. The relative decrease was greatest for the provision of swallow screen or assessment ( $\beta=-5.81 ; 95 \% \mathrm{Cl},-8.62$ to -2.99$)$, followed by the provision of antithrombotic medications within 48 hours of arrival $(\beta=-5.35 ; 95 \% \mathrm{Cl},-9.08$ to -1.61$)$ and discharge to inpatient rehabilitation ( $\beta=-2.44 ; 95 \% \mathrm{Cl}_{1}-3.75$ to -1.12$)$. There was no relative increase or decrease between groups observed in the proportion of patients mobilized during the admission.

Following the first week, the proportion provided a swallow screen or assessment increased for patients treated in stroke units and decreased for patients treated outside of stroke units during the pandemic period. Trends in the proportion of patients discharged to inpatient rehabilitation were unaffected in patients treated outside of stroke units, but there appeared to 


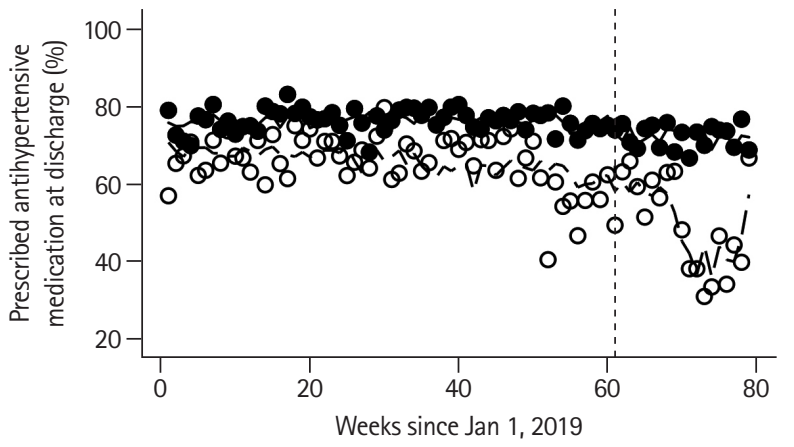

A

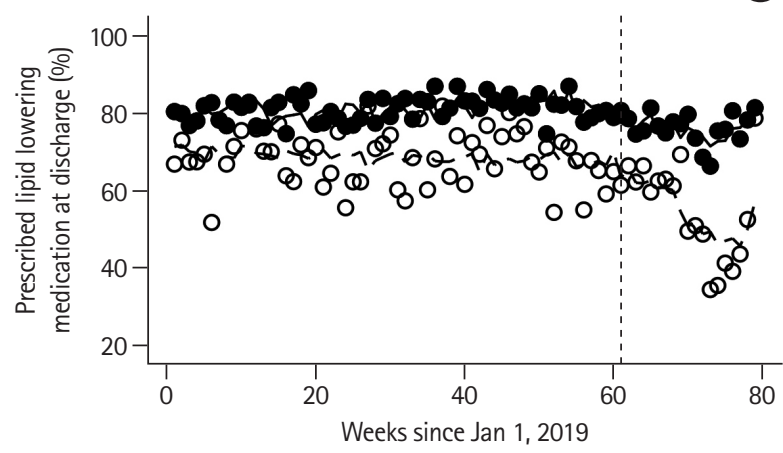

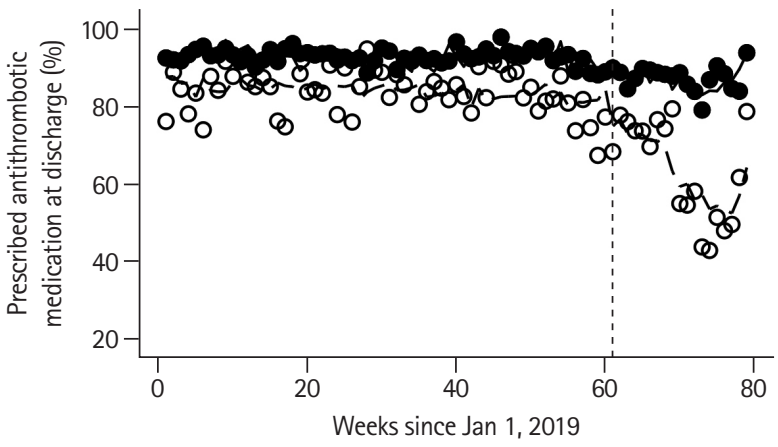

B

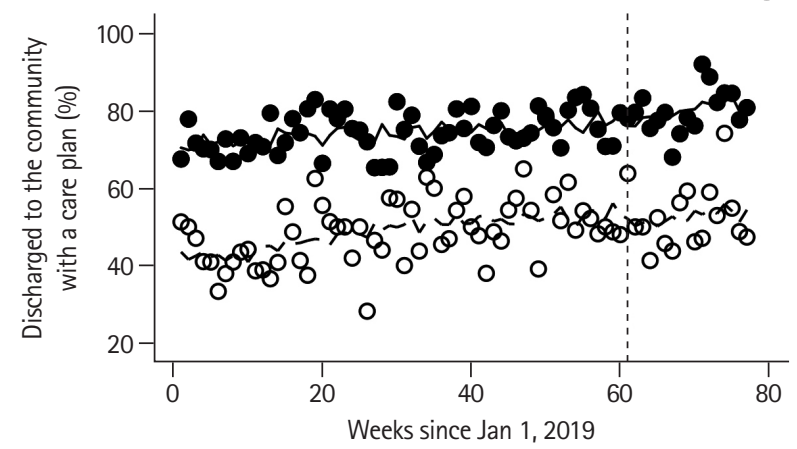

D

Treated in a stroke unit: Treated in other ward setting:

Actual $\longrightarrow$ Predicted

Actual - - - Predicted

Figure 2. Proportion of patients prescribed secondary prevention medications (A: antihypertensive medication; B: antithrombotic medication; C: lipid lowering medication) at discharge and (D) discharged to the community with a care plan. The vertical line (week 61) indicates the week of the interruption used in this study (1/3/2020, the first coronavirus disease 2019 [COVID-19] related death in Australia). Significant difference in weekly trend between groups prior to interruption ( $\beta$ and $95 \%$ confidence intervals [Cls] presented): discharged to the community with a care plan $-0.09(95 \% \mathrm{Cl},-0.17$ to -0.01$)$. Significant difference during week of interruption between groups (week 61): discharged to the community with a care plan 3.61 (95\% $\mathrm{Cl}, 0.28$ to 6.94$)$. Significant difference in weekly trend between groups in post-interruption period: provided antihypertensive medication at discharge $0.58(95 \% \mathrm{Cl}, 0.08$ to 1.07$)$; provided antithrombotic medication at discharge 1.08 ( $95 \% \mathrm{Cl}, 0.78$ to 1.38 ); provided lipid lowering medication at discharge 0.69 ( $95 \% \mathrm{Cl}, 0.28$ to 1.09$)$. $\beta$ in a period signifies the the difference in the average weekly percentage change over several weeks (trend) between groups. $\beta$ during the week of the interruption signifies the difference in a percentage change during that week between groups. Positive values indicate a greater relative percentage increase in the provision of evidence-based care in stroke units compared with other ward settings. Negative values indicate a greater relative percentage decrease in the provision of evidence-based care in stroke units compared with other ward settings. Other point estimates relevant to the figure are provided in the Supplementary Table 2.

be an increase in patients discharged to inpatient rehabilitation in those treated in stroke units. This corresponded to a $0.86 \%$ per week decrease in the proportion provided a swallow screen or assessment observed in patients treated in alternate ward settings relative to those treated in a stroke unit $(\beta=0.86$; $95 \% \mathrm{Cl}, 0.48$ to 1.24). There were similar findings for the proportion of patients discharged to inpatient rehabilitation, with a $0.52 \%$ per week decrease observed in patients treated in alternate ward settings relative to those treated in a stroke unit $(\beta=0.52 ; 95 \% \mathrm{Cl}, 0.09$ to 0.95$)$.

\section{Trends in the provision of secondary prevention medications and care plans}

In the pre-pandemic period, there was a small difference observed in the trends of provision of care plans at discharge be- tween patients treated in stroke units and those treated outside of stroke units (Figure 2, Supplementary Table 2). There was a $0.09 \%$ per week decrease in the provision of care plans among those discharged to the community in patients treated in stroke units relative to those treated outside of a stroke unit $(\beta=-0.09 ; 95 \% \mathrm{Cl},-0.17$ to -0.01$)$. No such differences in trends between groups were seen in the pre-pandemic period for the provision of secondary prevention medications at discharge.

In the first week of the pandemic period, the proportion of patients treated in stroke units that were discharged with a care plan increased relative to those treated outside of stroke units ( $\beta=3.61 ; 95 \% \mathrm{Cl}, 0.28$ to 6.94$)$. No such differences between groups were observed for the provision of secondary prevention medications at discharge. 
During the pandemic period, trends in the provision of secondary prevention medications were unaffected for patients treated in stroke units, but the proportions of patients treated in alternate ward settings who were provided secondary prevention medications decreased. There was a $0.58 \%$ per week decrease in the provision of antihypertensive medications at discharge observed in patients treated in alternate ward settings relative to those treated in a stroke unit $(\beta=0.58 ; 95 \% \mathrm{Cl}$, 0.08 to 1.07 ). There were similar findings for the provision of antithrombotic medications ( $\beta=1.08 ; 95 \% \mathrm{Cl}, 0.78$ to 1.38$)$ and lipid lowering medications ( $\beta=0.69 ; 95 \% \mathrm{Cl}, 0.28$ to 1.09 ). These differences in trends between groups were not seen for the provision of care plans in the pandemic period.

\section{Discussion}

To our knowledge, an in-depth assessment of the effect of reduced access to specialized stroke wards and clinicians as an unintended consequence of the COVID-19 pandemic has not been reported. Globally, there have been concerns about the effects of staff redeployment and the reorganization of stroke services, in addition to people with stroke symptoms delaying their presentation to the hospital..$^{10}$ Reports from other countries on the overall changes to the quality of care for patients with stroke have been mixed and mainly focused on use of thrombolysis or endovascular clot retrieval for those with stroke. ${ }^{11-14}$ The changes in the resources available for stroke in Australian hospitals has meant fewer patients getting access to stroke units during the pandemic. ${ }^{6}$ However, what we have learned in this investigation of care in stroke units compared with other ward settings is that the quality of care provided to patients treated in stroke units was largely unaffected. In contrast, with more patients being managed in alternate wards since the pandemic began, our findings of poorer quality of care in these settings when compared with before the pandemic is concerning. The findings reinforce the importance of ensuring the availability of doctors, nurses, and allied health staff with expertise and experience in managing patients with stroke are treating people with stroke. Care in stroke units by staff with expertise must be preferentially offered during a pandemic to ensure standards of care are upheld.

In this study, we examined evidence-based care that was undertaken in the wards following care in emergency departments. We found evidence that the quality of care may have been negatively affected by changes to hospital systems made in response to the COVID-19 pandemic. Using the same cohort, we had previously reported that access to intravenous thrombolysis and endovascular clot retrieval were similar; however, door-to-needle times were longer during the peak national pandemic period (March to April 2020). ${ }^{6}$

In addition, the overall proportion of patients with stroke or transient ischemic attack that received evidence-based therapies during their hospital admission had declined during the pandemic period. ${ }^{6}$ However, we did not understand the main driver for these findings until the analysis conducted for this current study. Consistent with prior research, we found evidence that stroke units were superior to alternate wards in providing evidence-based care. ${ }^{5,15}$ We also found novel evidence that the disparity in the provision of evidence-based therapies between patients treated in stroke units compared with those treated in other ward settings increased during the pandemic period, when compared with pre-pandemic standards. We surmise that competing priorities or lack of experience in how to manage different aspects of stroke in alternate wards has led to these findings.

The strengths of this study include the large standardized dataset of patients admitted with stroke and transient ischemic attack in major public hospitals treating stroke in five states and territories of Australia. In Australia, over a quarter of hospitals treating patients with stroke do not have a stroke unit. ${ }^{16}$ All hospitals included in this analysis had a stroke unit. Another strength is that interrupted time series methods were used, with seasonality considered and time-varying confounders included. Interrupted time series methods are being increasingly used and are considered a robust tool for assessing changes resulting from health interventions..$^{17,18}$

Owing to the COVID-19 pandemic, clinicians working in hospitals that participate in the AuSCR have reported that one in 10 had their bed numbers reduced in the stroke unit, one in four their stroke unit moved, and one in three stroke units had their staff redeployed to other duties. ${ }^{6}$ This may explain why fewer patients accessed stroke units during the pandemic periods examined. However, one limitation is that we do not know whether patients with stroke had COVID-19 and may have required management in a COVID-19 ward. Notably, approximately $1.5 \%$ of people with COVID-19 have been found to ex-

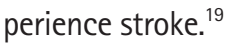

Another limitation of our study is that we did not have data available on treatment and outcomes after discharge from the hospital. Therefore, we were unable to investigate if care usually provided during the hospital admission or at discharge was substituted with other health services. Whether the poorer quality of care provided during the admission was observed during the pandemic period has resulted in poorer quality of life and greater mortality will be investigated in future research. 


\section{Conclusions}

Stroke is a leading cause of death ${ }^{20}$ and disability, ${ }^{21}$ and it is essential that patients continue to be treated in stroke units, as treatment in a stroke unit reduces the number of people with poor outcomes. ${ }^{4,5}$ Our findings underscore the importance of why we need to treat all patients with stroke in specialized wards in line with national guideline recommendations, regardless of whether we are in the midst of a pandemic.

\section{Supplementary materials}

Supplementary materials related to this article can be found online at https://doi.org/10.5853/jos.2021.02530.

\section{Disclosure}

The authors have no financial conflicts of interest.

\section{Acknowledgments}

We acknowledge Prof. Helen Dewey, Prof. Mark Parsons, Ms. Jot Ghuliani, and other members of the AuSCR office team, governance committees, and data contributors to the AuSCR (see Supplementary acknowledgments).

The Australian Stroke Clinical Registry was funded from various grants obtained from Stroke Foundation, Queensland Health, Victorian government, Tasmania Government, South Australian government. The following authors received Research Fellowship support from the National Health and Medical Research Council: Dominique A. Cadilhac (1154273); and the National Heart Foundation of Australia: Natasha A. Lannin (102055).

\section{References}

1. McCabe R, Schmit N, Christen P, D'Aeth JC, Løchen A, Rizmie $D$, et al. Adapting hospital capacity to meet changing demands during the COVID-19 pandemic. BMCMed 2020;18:329.

2. Brainin M. Stroke care and the COVID19 pandemic: words from our president. World Stroke Organization. https://www. world-stroke.org/news-and-blog/news/stroke-care-and-thecovid19-pandemic. 2020. Accessed September 22, 2021.

3. Coote S, Cadilhac DA, O'Brien E, Middleton S; Acute Stroke Nurses Education Network (ASNEN) Steering Committee. Letter to the editor regarding: critical considerations for stroke management during COVID-19 pandemic in response to Inglis et al., Heart Lung Circ. 2020;29(9):1263-1267. Heart
Lung Circ 2020;29:1895-1896.

4. Stroke Unit Trialists' Collaboration. Organised inpatient (stroke unit) care for stroke. Cochrane Database Syst Rev 2013;2013:CD000197.

5. Cadilhac DA, Andrew NE, Lannin NA, Middleton S, Levi CR, Dewey HM, et al. Quality of acute care and long-term quality of life and survival: the Australian Stroke Clinical Registry. Stroke 2017;48:1026-1032.

6. Cadilhac DA, Kim J, Tod EK, Morrison JL, Breen SJ, Jaques K, et al. COVID-19 pandemic impact on care for stroke in Australia: emerging evidence from the Australian Stroke Clinical Registry. Front Neurol 2021;12:621495.

7. Cadilhac DA, Lannin NA, Anderson CS, Levi CR, Faux S, Price $C$, et al. Protocol and pilot data for establishing the Australian Stroke Clinical Registry. Int J Stroke 2010;5:217-226.

8. Tu JV, Willison DJ, Silver FL, Fang J, Richards JA, Laupacis A, et al. Impracticability of informed consent in the Registry of the Canadian Stroke Network. N Engl J Med 2004;350:14141421.

9. Linden A, Arbor A. Conducting interrupted time-series analysis for single- and multiple-group comparisons. Stata J 2015;15:480-500.

10. Markus HS, Brainin M. COVID-19 and stroke: a global World Stroke Organization perspective. Int J Stroke 2020;15:361-364.

11. Chen $\mathrm{CH}_{\text {, Liu } \mathrm{CH}}$, Chi NF, Sung PS, Hsieh CY, Lee M, et al. Maintenance of stroke care quality amid the coronavirus disease 2019 outbreak in Taiwan. J Stroke 2020;22:407-411.

12. Desai SM, Guyette FX, Martin-Gill C, Jadhav AP. Collateral damage: impact of a pandemic on stroke emergency services. J Stroke Cerebrovasc Dis 2020;29:104988.

13. Hoyer $C$, Ebert $A$, Huttner HB, Puetz V, Kallmünzer B, Barlinn $K_{1}$ et al. Acute stroke in times of the COVID-19 pandemic: a multicenter study. Stroke 2020;51:2224-2227.

14. Rudilosso $\mathrm{S}$, Laredo $\mathrm{C}$, Vera V, Vargas $\mathrm{M}$, Renú $\mathrm{A}$, Llull L, et al. Acute stroke care is at risk in the era of COVID-19: experience at a comprehensive stroke center in Barcelona. Stroke 2020:51:1991-1995.

15. Cadilhac DA, Ibrahim J, Pearce DC, Ogden KJ, McNeill J, Davis SM, et al. Multicenter comparison of processes of care between stroke units and conventional care wards in Australia. Stroke 2004;35:1035-1040.

16. Stroke Foundation. National Stroke Audit Acute Services Report 2019. Melbourne: Stroke Foundation, 2019

17. Bernal JL, Cummins S, Gasparrini A. Interrupted time series regression for the evaluation of public health interventions: a tutorial. Int J Epidemiol 2017;46:348-355.

18. Schaffer AL, Dobbins TA, Pearson SA. Interrupted time series analysis using autoregressive integrated moving average 
(ARIMA) models: a guide for evaluating large-scale health interventions. BMC Med Res Methodo/ 2021;21:58.

19. Merkler AE, Parikh NS, Mir S, Gupta A, Kamel H, Lin E, et al. Risk of ischemic stroke in patients with coronavirus disease 2019 (COVID-19) vs patients with influenza. JAMA Neurol 2020;77:1-7.

20. Stroke Foundation. Clinical Guidelines for Stroke Management. Stroke Foundation. 2017. https://informme.org.au/en/
Guidelines/Clinical-Guidelines-for-Stroke-Management. Assessed October 12, 2021.

21. Krishnamurthi RV, Ikeda T, Feigin VL. Global, regional and country-specific burden of ischaemic stroke, intracerebral haemorrhage and subarachnoid haemorrhage: a systematic analysis of the global burden of disease study 2017. Neuroepidemiology 2020;54:171-179. 
Supplementary Table 1. Differences in the trends of provision of nursing and allied health interventions between stroke units and other ward settings

\begin{tabular}{lccc}
\hline & $\begin{array}{c}\text { Difference in the weekly } \\
\text { trend prior to the interruption }\end{array}$ & $\begin{array}{c}\text { Difference during the week } \\
\text { of the interruption }\end{array}$ & $\begin{array}{c}\text { Difference in the weekly } \\
\text { trend in the post-interruption period }\end{array}$ \\
\hline $\begin{array}{l}\text { Provided antithrombotic medication } \\
\text { within } 48 \text { hours of arrival* }\end{array}$ & $0.09(0.04 \text { to } 0.14)^{+}$ & $-5.35(-9.08 \text { to }-1.61)^{+}$ & $0.19(-0.25$ to 0.63$)$ \\
$\begin{array}{l}\text { Mobilized during the admission } \\
\begin{array}{l}\text { Provided swallow screen } \\
\text { or assessment }\end{array}\end{array}$ & $-0.15(-0.23 \text { to }-0.07)^{+}$ & $2.88(-0.34$ to 6.11$)$ & $0.06(-0.62$ to 0.75$)$ \\
$\begin{array}{l}\text { Discharged to inpatient } \\
\text { rehabilitation }\end{array}$ & $-0.01(-0.04$ to 0.02$)$ & $-5.81(-8.62 \text { to }-2.99)^{+}$ & $0.86(0.48 \text { to } 1.24)^{+}$ \\
\hline
\end{tabular}

Values are presented as $\beta$ (95\% confidence interval). $\beta$ In a period signifies the difference in the average weekly percentage change over several weeks (trend) between groups. $\beta$ During the week of the interruption signifies the difference in percentage change during that week between the groups. Positive values indicate a greater relative percentage increase in the provision of evidence-based care in stroke units compared with other ward settings. Negative values indicate a greater relative percentage decrease in the provision of evidence-based care in stroke units compared with other ward settings.

*Excluding patients with intracerebral hemorrhage; ${ }^{+}$Values indicate significant differences.

Supplementary Table 2. Differences in the trends of provision of secondary prevention medications and care plans at discharge between stroke units and other ward settings

\begin{tabular}{lccc}
\hline & $\begin{array}{c}\text { Difference in the weekly } \\
\text { trend prior to the interruption }\end{array}$ & $\begin{array}{c}\text { Difference during the week } \\
\text { of the interruption }\end{array}$ & $\begin{array}{c}\text { Difference in the weekly } \\
\text { trend in the post-interruption period }\end{array}$ \\
\hline $\begin{array}{l}\text { Prescribed antihypertensive } \\
\text { medication at discharge }\end{array}$ & $0.09(-0.05$ to 0.22$)$ & $-2.82(-8.31$ to 2.67$)$ & $0.58(0.08 \text { to } 1.07)^{+}$ \\
$\begin{array}{c}\text { Prescribed antithrombotic } \\
\text { medication at discharge }\end{array}$ & $0.01(-0.08$ to 0.1$)$ & $-3.49(-7.76$ to 0.78$)$ & $1.08(0.78 \text { to } 1.38)^{+}$ \\
$\begin{array}{c}\text { Prescribed lipid lowering } \\
\text { medication at discharge }\end{array}$ & $0.05(-0.02$ to 0.13$)$ & $3.61(0.28 \text { to } 6.94)^{+}$ & $0.69(0.28 \text { to } 1.09)^{+}$ \\
$\begin{array}{c}\text { Discharged to the community } \\
\text { with a care plan }\end{array}$ & $-0.09(-0.17 \text { to }-0.01)^{+}$ & $0.35(-0.64$ to 1.34$)$ \\
\hline
\end{tabular}

Values are presented as $\beta$ (95\% confidence interval). $\beta$ In a period signifies the difference in the average weekly percentage change over several weeks (trend) between groups. $\beta$ During the week of the interruption signifies the difference in percentage change during that week between the groups. Positive values indicate a greater relative percentage increase in the provision of evidence-based care in stroke units compared with other ward settings. Negative values indicate a greater relative percentage decrease in the provision of evidence-based care in stroke units compared with other ward settings.

*Excluding patients with intracerebral hemorrhage; ' ${ }^{*}$ alues indicate significant differences. 


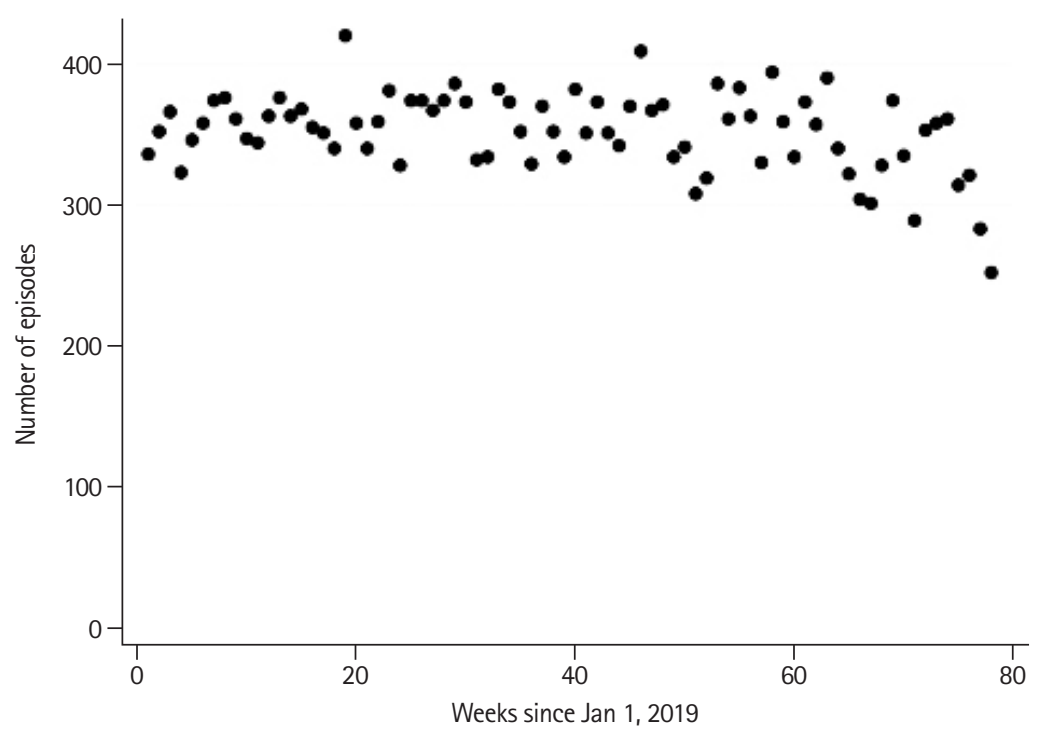

Supplementary Figure 1. Number of admissions per week. Mean number of admissions per week: 348.2532 (standard deviation: 45.08808). 


\section{Supplementary acknowledgments}

\section{AuSCR COVID-19 reporting consortium group}

The following authors contributed to the interpretation of data, critical revision of the article, and collection of data.

Alaa Alghamry (The Prince Charles Hospital, Queensland, Australia)

Lauren Arthurson (Echuca Regional Health, Victoria, Australia)

Jonnel Boco (Caboolture, Queensland, Australia)

Melanie Brown (Goulburn Valley Health, Victoria, Australia)

Helen Brown (Princess Alexandra Hospital, Queensland, Australia)

Ernie Butler (Peninsula Health, Victoria, Australia)

Greg Cadigan (Queensland Health, Queensland, Australia)

Anna Clissold (South West Healthcare, Victoria, Australia)

Janell Cole (North West Regional Hospital, Tasmania, Australia)

Douglas Crompton (Northern Health, Victoria, Australia)

Vanessa Crosby (Albury Wodonga Health, Victoria \& New South

Wales, Australia)

Geoffrey Donnan (University of Melbourne, Parkville, Australia)

Angela Dos Santos (Alfred Health, Victoria, Australia)

Ramesh Durairaj (Cairns Hospital, Queensland, Australia)

Chris Ebersohn (Wimmera Base Hospital, Victoria, Australia)

Andrew Evans (Westmead Hospital, New South Wales, Australia)

Brett Forge (West Gippsland Hospital, Victoria, Australia)

Karen Fuller (Wollongong Hospital, New South Wales, Australia)

Nisal Gange (Toowoomba Hospital, Queensland, Australia)

Yash Gawarikar (Calvary Hospital, Australian Capital Territory,

Australia)

Richard Geraghty (Redcliffe Hospital, Queensland, Australia)

Rohan Grimley (Sunshine Coast University Hospital, Birtinya, Queensland)

Susan Hillier (University of South Australia, Adelaide, South Australia, Australia)

Kate Jackson (Agency for Clinical Innovation, New South Wales, Australia)

Martin Jude (Wagga Wagga Hospital, New South Wales, Australia)

Salman Khan (Nepean Hospital, New South Wales, Australia)

Monique Kilkenny (Monash University, Victoria, Australia)

Thomas Kraemer (Ballarat Base Services, Victoria, Australia)

Alex Lau (Logan Hospital, Queensland, Australia)

Henry Ma (Monash Health, Victoria, Australia)

Krishna Mandaleson (Central Gippsland Health Service)

Romesh Markus (St Vincent's Hospital, Sydney, New South

Wales, Australia)

Peter Mitchell (Royal Melbourne Hospital, Victoria, Australia)

Stephen Moore (Lismore Base Hospital, New South Wales, Australia)
Julie Morrison (The Florey Institute of Neuroscience and Mental Health, Victoria, Australia)

Neha Nandal (Mackay Base Hospital, Queensland, Australia)

Kim Parrey (Port Macquarie Hospital, New South Wales, Australia)

Lauren Pesavento (Melbourne Health, Victoria, Australia)

Juan Rois-Gnecco (Ipswich, Queensland, Australia)

Darshan Shah (Gold Coast University Hospital, Queensland, Australia)

Daniel Schweitzer (Mater Hospital Brisbane, Queensland, Australia)

Amanda Siller (Queen Elizabeth II Jubilee Hospital, Queensland, Australia)

Jenni Steel (Port Macquarie Hospital, New South Wales, Australia)

Karen Stephens (Eastern Health, Victoria, Australia)

Louise Starkie (Hamilton Base Hospital, Victoria, Australia)

Meng Tan (Gold Coast University Hospital and Robina Hospital,

Queensland, Australia)

Vincent Thijs (Austin Health, Victoria, Australia)

Dinesh Tryambake (Launceston General Hospital, Tasmania, Australia)

Rebecca Weir (Northeast Health Wangaratta, Victoria, Australia) Leanne Whiley (Rockhampton Hospital, Queensland, Australia) Richard White (Townsville Hospital, Queensland, Australia)

Tissa Wijeratne (Western Health, Victoria, Australia)

Matt Willcourt (Flinders Medical Centre, South Australia, Australia)

Nigel Wolfe (Blacktown Hospital, New South Wales, Australia)

Andrew Wong (Royal Brisbane and Women's Hospital, University of Queensland, Queensland, Australia)

Peter Wood (Bundaberg Hospital \& Hervey Bay Hospital, Queensland, Australia)

Jorge Zavala (Alfred Health, Victoria, Australia).

\section{The AuSCR consortium group}

Evelyn Anino (Queen Elizabeth II Jubilee Hospital, Queensland, Australia)

Joanne Ashford (Mackay Hospital, Queensland, Australia)

Jennifer Bergqvist (Western Health, Victoria, Australia)

Haylee Berrill (Gold Coast University \& Robina Hospitals, Queensland, Australia)

Pauline Blaney (Hervey Bay Hospital, Queensland, Australia)

Deirdre Broadby (Royal Hobart Hospital, Tasmania, Australia)

Michelle Bronca (Flinders Medical Centre, South Australia, Australia)

Camelia Burdusel (Blacktown Hospital, New South Wales, Australia) 
Carla Borg Caruana (Alfred Health, Victoria, Australia)

Aylissa Canning (Princess Alexandra Hospital, Queensland, Australia)

Kristine Caprecho (Calvary Hospital, Australian Capital Territory, Australia)

John Carmody (Wollongong Hospital, New South Wales, Australia)

Shaji Chacko (Gympie Hospital, Queensland, Australia)

Kerri Chamberlain (Wimmera Base Hospital, Victoria, Australia)

Michelle Coad (Port Macquarie Hospital, New South Wales, Australia)

Kathryn Colebourne (The Prince Charles Hospital, Queensland, Australia)

Tessa Coupland (Bendigo Health, Victoria, Australia)

Shahla Cowans (Canberra Health Services, Australian Capital Territory, Australia)

Dijana Cukanovic-Krebs (Cairns Hospital, Queensland, Australia)

Sonia Dann (Bundaberg Hospital, Queensland, Australia)

Lizzie Dodd (Royal Adelaide Hospital, South Australia, Australia)

Helen Eaves (Bundaberg Hospital, Queensland, Australia)

Linda Edwards (Ipswich Hospital, Queensland, Australia)

Troy Elliott (Cairns Hospital, Queensland, Australia)

Tanya Frost (Eastern Health, Victoria, Australia)

Karen Fuller (Wollongong Hospital, New South Wales, Australia)

Renae Gamble (Austin Health, Victoria, Australia)

Belinda Green (Alfred Health, Victoria, Australia)

Patrick Groot (South West Healthcare, Victoria, Australia)

Kushantha Gunarathne (Barinsdale Regional health Service,

Victoria, Australia)

Casey Hair (Ballarat Base Services, Victoria, Australia

Nicola Hall (Logan Hospital, Queensland, Australia)

Emma Harding (Swan Hill District Health, Victoria, Australia)

Carolyn Harrison (Launceston General Hospital, Tasmania, Australia)

Kim Hoffman (Lismore Base Hospital, New South Wales, Australia

Marnie Hollywood (Caboolture Hospital, Queensland, Australia)

Deborah Holmes (St Vincent's Hospital, Sydney, New South

Wales, Australia)

Anne Hooper (Mackay Hospital, Queensland, Australia)

Tracey Howlett (Logan Hospital, Queensland, Australia)

Karen Hudson (Gympie Hospital, Queensland, Australia)

Michelle Hutchinson (Flinders Medical Centre, South Australia,

Australia)

Manju John (Canberra Health Services, Australian Capital Terri-

tory, Australia)

Cath Jones (Bass Coast Health, Victoria, Australia)

Tracey Johnson (Gold Coast University Hospital, Queensland, Australia)
Caitlin Kearney (The Prince Charles Hospital, Queensland, Australia)

Lorraine Keene (West Gippsland Hospital, Victoria, Australia)

Kanaga Lagma (Peninsula Health, Victoria, Australia)

Kylie Lodge (Queen Elizabeth II Jubilee Hospital, Queensland,

Australia)

Jodi Lynch (Monash Health, Victoria, Australia)

Laura Macdonald (Bairnsdale Regional health Service, Victoria, Australia)

Elizabeth Mackey (Western Health, Victoria, Australia)

Lyn Malone (Northeast Health Wangaratta, Victoria, Australia)

Janet May (Latrobe Regional Hospital, Victoria, Australia)

Marie McCaig (Mater Hospital, Queensland, Australia)

Amanda McKee (Princess Alexandra Hospital, Queensland, Australia)

lan Meade (Townsville Hospital, Queensland, Australia)

Jessica Miller (Monash Health, Victoria, Australia)

Katherine Mohr (Wagga Wagga Hospital, New South Wales, Australia)

Maxine Munting (North West Regional Hospital, Tasmania, Australia)

Ashley Murray (West Gippsland Hospital, Victoria, Australia)

Linda Norrie (Townsville Hospital, Queensland, Australia)

Trish Oxley (Swan Hill District Health, Victoria, Australia)

Kirsty Page (St Vincent's Hospital, Sydney, New South Wales, Australia)

Arun Raju (Toowoomba Hospital, Queensland, Australia)

Emara Ramchander (Western Health, Victoria, Australia)

Erin Ray (Bendigo Health, Victoria, Australia)

Deidre Rennick (Wimmera Base Hospital, Victoria, Australia)

Timothy Richardson (Toowoomba Hospital, Queensland, Australia)

Nina Roberts (Wimmera Base Hospital, Victoria, Australia)

Ros Roberts (Mildura Base Public Hospital, Victoria, Australia)

Anne Rodda (Northern Health, Victoria, Australia)

Simone Rogers (Bundaberg Hospital, Queensland, Australia)

David Rosaia (Bendigo Health, Victoria, Australia)

Donna Rowley (Sunshine Coast University Hospital, Queensland, Australia)

Ramesh Sahathevan (Ballarat Base Services, Victoria, Australia)

Sebastian Saj (Nepean Hospital, New South Wales, Australia)

Lauren Sanders (St Vincent's Hospital, Victoria, Australia)

Mark Savage (Bendigo Health, Victoria, Australia)

Patrick Scarff (St Vincent's Hospital, Victoria, Australia)

Betsy Shaju (Ipswich Hospital, Queensland, Australia)

Heather Smith (University Hospital Geelong, Victoria, Australia)

Jennifer Steel (Port Macquarie Hospital, New South Wales, Australia)

Margaret Stevenson (Peninsula Health, Victoria, Australia) 
Leanne Taylor (Wimmera Base Hospital, Victoria, Australia) Smisha Thomas (Royal Melbourne Hospital, Victoria, Australia) Anne Van Berkel (Central Gippsland Health Service, Victoria, Australia)

Tamara Wanklyn (Western Health, Victoria, Australia) Jaqueline Watson (Westmead Hospital, New South Wales, Australia)

Michelle Webb (Royal Brisbane and Women's Hospital, Queensland, Australia)

Tracey Whitehead (Townsville Hospital, Queensland, Australia)

Tanya Williams (Redcliffe Hospital, Queensland, Australia)

Jerry Wong (Queen Elizabeth II Jubilee Hospital, Queensland, Australia)

Toni Wonson (Wollongong Hospital, New South Wales, Australia) Melissa Wood (Royal Brisbane and Women's Hospital, Queensland, Australia)

Martin Zolfel (Nepean Hospital, New South Wales, Australia)

\section{AuSCR office staff (not listed elsewhere)}

Helen Carter (The Florey Institute of Neuroscience and Mental Health, Victoria, Australia)

Adele Gibbs (The Florey Institute of Neuroscience and Mental Health, Victoria, Australia)

Shaun Hancock (The Florey Institute of Neuroscience and Mental Health, Victoria, Australia)

Marcus Lester (The Florey Institute of Neuroscience and Mental Health, Victoria, Australia)

Violet Marion (The Florey Institute of Neuroscience and Mental Health, Victoria, Australia)

Karen Moss (The Florey Institute of Neuroscience and Mental Health, Victoria, Australia)

Kate Paice (The Florey Institute of Neuroscience and Mental Health, Victoria, Australia)

Nancy Pompeani (The Florey Institute of Neuroscience and Mental Health, Victoria, Australia)

Olivia Ryan (The Florey Institute of Neuroscience and Mental Health, Victoria, Australia)

Sam Shehata (The Florey Institute of Neuroscience and Mental Health, Victoria, Australia)

Claire Weickhardt (The Florey Institute of Neuroscience and Mental Health, Victoria, Australia)

AuSCR committee members (not listed elsewhere) Julie Bernhardt (The Florey Institute of Neuroscience and Men- tal Health, Victoria, Australia)

Chris Bladin (The Florey Institute of Neuroscience and Mental Health, Victoria, Australia)

Ken Butcher (Prince of Wales Hospital, New South Wales, Australia)

Andrew Cheung (Prince of Wales and Liverpool Hospitals, New South Wales, Australia)

Philip Choi (Eastern Health, Victoria, Australia)

Alan Coulthard (Royal Brisbane and Womens Hospital, Queensland, Australia)

Coralie English (Hunter Medical Research Institute, New South Wales, Australia)

Sue Evans (Monash University, Victoria, Australia)

Darshan Ghia (Fiona Stanley Hospital, Western Australia, Australia)

Erin Godecke (Edith Cowan University, Western Australia, Australia)

Martin Krause (Royal North Shore Hospital, New South Wales, Australia)

Peter Hand (Alfred Health, Victoria, Australia)

Suzanne Kuys (Australian Catholic University, Queensland, Australia)

Mark Mackay (Royal Children's Hospital, Victoria, Australia)

John McNeil (Monash University, Victoria, Australia)

Ferdi Miteff (Royal North Shore Hospital, New South Wales, Australia)

Jennifer Muller (Consumer representative, Queensland, Australia) Lisa Murphy (Stroke Foundation, Melbourne, Victoria, Australia) Michael Pollack (Hunter Stroke Service, New South Wales, Australia)

Rebecca Scroop (Royal Adelaide Hospital, South Australia, Australia)

Mark Simcocks (Consumer representative, Victoria, Australia)

Frances Simmonds (University of Wollongong, New South Wales, Australia)

Brendan Steinfort (Royal North Shore Hospital, New South Wales, Australia)

Belinda Stojanovski (Royal Children's Hosptial, Parkville

Amanda Thrift (Monash University, Victoria, Australia)

Jason Wenderoth (Prince of Wales Hospital, New South Wales, Australia)

Andrew Wesseldine (St John of God Hospital, Western Australia, Australia) 